\title{
SCHUR INDICES AND THE GALOIS GROUP
}

\author{
EUGENE SPIEGEL
}

\begin{abstract}
In this note we show that the order of the Schur index of an irreducible representation divides the order of a certain subgroup of the Galois group of a cyclotomic extension of the ground field.
\end{abstract}

For $\boldsymbol{F}$ a field, $\boldsymbol{G}$ a finite group of exponent $\boldsymbol{n}$ and $\chi$ an irreducible character of $\boldsymbol{G}$, we let $m_{F}(\chi)$ denote the Schur index of $\chi$ over $F$. A famous theorem of Brauer states that $m_{F}(\chi)=1$ if $\zeta_{n} \in F$ where $\zeta_{n}$ denotes a primitive $n$th root of unity. In 1975, Goldschmidt and Isaacs [3] showed that $p \nmid m_{F}(x)$ if $p$ is a prime with the property that the $p$-Sylow subgroup of $\operatorname{Gal}\left(F\left(\zeta_{n}\right) \mid F\right)$ is cyclic, except possibly when $p=2$ and $\sqrt{-1} \notin F$. Fein [2] showed that the exceptional case of the previous theorem cannot hold if -1 is a sum of two squares in $F$.

In this note we strengthen the previous results by showing

THEOREM. Let $F$ be a field of characteristic $0, n$ a positive integer and $\operatorname{Gal}\left(F\left(\zeta_{n}\right) \mid F\right)$ $\simeq H_{1} \times H_{2} \times \cdots \times H_{k}$ with $H_{i}$ cyclic and $\left|H_{i}\right||| H_{i-1} \mid, i=2, \ldots, k$. Let $G$ be $a$ finite group of exponent $n$ and $\chi$ an irreducible character of $G$. Then

$$
m_{F}(\chi)|| H_{2} \mid \quad \text { if } m_{F}(\chi) \neq 2(\bmod 4)
$$

and

$$
m_{F}(\chi)|2| H_{2} \mid \quad \text { if } m_{F}(\chi) \neq 2(\bmod 4) .
$$

Before proving the theorem, we need an elementary lemma.

Lemma. Suppose $T=T_{1} \times T_{2} \times \cdots \times T_{k}$ with $T_{i}$ finite cyclic groups and $\left|T_{i}\right||| T_{i-1} \mid, i=2, \ldots, k$. Let $a, b \in T$ each be of order $r$ with $\langle a\rangle \cap\langle b\rangle=\{e\}$ $(\langle a\rangle$ is the group generated by $a)$. Then $r|| T_{2} \mid$.

Proof. Write $H=T_{2} \times \cdots \times T_{k}$. Then $\left|T_{2}\right|$ is the exponent of $H$. Write $a=\left(t_{1}, h_{1}\right), b=\left(t_{2}, h_{2}\right)$ with $t_{i} \in T_{1}, h_{i} \in H$. Let $s_{i}=\left|\left\langle h_{i}\right\rangle\right|, i=1,2$. Then $s_{i} \mid r$ and $a^{s_{1}}=\left(t_{1}^{s_{1}}, e\right), b^{s_{2}}=\left(t_{2}^{s_{2}}, e\right)$. As $\langle a\rangle \cap\langle b\rangle=\{e\}, \quad\left(\left|\left\langle a^{s_{1}}\right\rangle\right|,\left|\left\langle b^{s_{2}}\right\rangle\right|\right)=1$ and $\left(r / s_{1}, r / s_{2}\right)=1$. Thus $\left(s_{2} r, s_{1} r\right)=s_{1} s_{2}$. But $\left(s_{2} r, s_{1} r\right)=r\left(s_{1}, s_{2}\right)$, so $r=s_{1} s_{2} /\left(s_{1}, s_{2}\right)$ and $r$ is the least common multiple of $s_{1}$ and $s_{2}$. Hence $H$ contains an element of order $r$ and $r|| T_{2} \mid$.

Proof of THeORem. Let $L=Q\left(\zeta_{n}\right) \cap F$. Then $L$ is a finite extension of $Q$ and $\operatorname{Gal}\left(L\left(\zeta_{n}\right) \mid L\right) \simeq \operatorname{Gal}\left(F\left(\zeta_{n}\right) \mid F\right)$. But $F$ an extension of $L$ implies $m_{F}(\chi) \mid m_{L}(\chi)$ and so it is sufficient to show that $m_{L}(\chi)|| H_{2} \mid$, and we henceforth suppose that $F$ is a finite extension of $Q$.

Received by the editors April 15, 1980.

1980 Mathematics Subject Classification. Primary 20 C05. 
Suppose now that $q^{s} \| m_{F}(\chi)$ with $q$ a prime. Then there is a rational prime $p$ and a prime $P$ lying over $p$ in $F$ so that localization at $P$ gives $q^{s} \| m_{F_{P}}(\chi)$. Assume that $q^{s} \neq 2$ or $p \neq 2$.

Let $\phi: \operatorname{Gal}\left(F_{P}\left(\zeta_{n}\right) \mid F_{P}\right) \rightarrow \operatorname{Gal}\left(F\left(\zeta_{n}\right) \mid F\right)$ given by $\phi(\sigma)=\left.\sigma\right|_{F\left(\zeta_{n}\right)}$ for $\sigma \in$ $\operatorname{Gal}\left(F_{P}\left(\zeta_{n}\right) \mid F_{P}\right), \phi$ is an injection. Write $n=p^{e} t$ with $(t, p)=1$. Then $F_{P}\left(\zeta_{t}\right)$ is a cyclic extension of $F_{P}$ since it is a totally unramified extension. If $p=2$, the Schur subgroup of $F_{P}$ has at most two elements [5, Theorem 5.5] and so $q^{s} \neq 2$ or $p \neq 2$ implies $p \neq 2$ and $F_{P}\left(\zeta_{p} e\right)$ is a cyclic extension of $F_{P}$. Because $F_{P}\left(\zeta_{n}\right)=F_{P}\left(\zeta_{p} e, \zeta_{t}\right)$, $\operatorname{Gal}\left(F_{P}\left(\zeta_{n}\right) \mid F_{P}\right)$ is generated by two elements and thus so is the subgroup $\operatorname{Gal}\left(F_{P}\left(\zeta_{n}\right) \mid L\right)$ where $L=F_{P}(\chi) \subset F_{P}\left(\zeta_{n}\right)$. We can now write $\operatorname{Gal}\left(F_{P}\left(\zeta_{n}\right) \mid L\right)$ as the internal direct product of two cyclic groups $A_{1}$ and $A_{2}$. Let $S_{i}$ be the fixed field of $\boldsymbol{A}_{i}$.

We now show that $q^{s}|| A_{i} \mid$ for $i=1$, 2. If not, we can suppose without loss of generality, that $q^{u} \|\left|A_{1}\right|$ with $0 \leqslant u<s$.

Since $\operatorname{deg}\left(S_{2} \mid L\right)=A_{1}$, by Theorems 4.21 and 9.23 of Albert [1], $q^{s-u} \| m_{S_{2}}(\chi)$. But $S_{2}\left(\zeta_{n}\right)=F_{P}\left(\zeta_{n}\right)$ and $\operatorname{Gal}\left(S_{2}\left(\zeta_{n}\right) \mid S_{2}\right) \simeq A_{2}$ is cyclic. If $q$ is odd, by the theorem of Goldschmidt and Isaacs [3], $q \nmid m_{S_{2}}(\chi)$ giving a contradiction. If $q=2$ and $p \neq 2$ then -1 is a sum of two squares [4, Lemma 2.2], in $Q_{P} \subset F_{P}$ and so by the theorem of Fein [2], $q \nmid m_{S_{2}}(\chi)$ again giving our contradiction. Hence we can assume $q^{s}|| A_{i} \mid$ for $i=1,2$.

Let $\sigma_{i} \subset A_{i}$ for $i=1,2$ be elements of order $q^{s} .\left\langle\sigma_{1}\right\rangle \cap\left\langle\sigma_{2}\right\rangle=\{e\}$ since $A_{1} \cap A_{2}=\{e\}$. Then $\phi\left(\sigma_{1}\right)$ and $\phi\left(\sigma_{2}\right)$ are again elements in $\operatorname{Gal}\left(F\left(\zeta_{n}\right) \mid F\right)$ of order $q^{s}$ with $\left\langle\phi\left(\sigma_{1}\right)\right\rangle \cap\left\langle\phi\left(\sigma_{2}\right)\right\rangle=\{e\}$. By the lemma $q^{s}|| H_{2} \mid$ and the theorem is established.

We remark that the proof shows that $q^{s}|| H_{2} \mid$ unless $q^{s}=2$ and $p=2$.

\section{REFERENCES}

1. A. A. Albert, Structure of algebras, Amer. Math. Soc. Colloq. Publ., vol. 24, Amer. Math. Soc., Providence, R. I., 1961.

2. B. Fein, Schur indices and sums of squares, Proc. Amer. Math. Soc. 51 (1975), 31-34.

3. D. Goldschmidt and I. Isaacs, Schur indices in finite groups, J. Algebra 33 (1975), 191-199.

4. E. Spiegel and A. Trojan, On semi-simple group algebras. II, Pacific J. Math. 66 (1976), 553-559.

5. T. Yamada, The Schur subgroup of the Brauer group, Lecture Notes in Math., vol. 397, SpringerVerlag, Berlin and New York, 1974.

Department of Mathematics, University of Connecticut, Storrs, Connecticut 06268 